\title{
Effects of Stellar-Mass Black Holes on Massive Star Cluster Evolution
}

\author{
Sourav Chatterjee ${ }^{1}$, Meagan Morscher ${ }^{1}$, Carl L. Rodriguez ${ }^{1}$, Bharat \\ Pattabiraman $^{1}$ and Frederic A. Rasio ${ }^{1}$ \\ ${ }^{1}$ Center for Interdisciplinary Exploration and Research in Astrophysics \\ Northwestern University \\ 2145 Sheridan Road, Evanston, IL 60208, USA \\ email: sourav.chatterjee@northwestern.edu
}

\begin{abstract}
Recent observations have revealed the existence of stellar mass black hole (BH) candidates in some globular clusters (GC) in the Milky Way and in other galaxies. Given that the detection of BHs is challenging, these detections likely indicate the existence of large populations of BHs in these clusters. This is in direct contrast to the past understanding that at most a handful of BHs may remain in old GCs due to quick mass segregation and rapid mutual dynamical ejection. Modern realistic star-by-star numerical simulations suggest that the retention fraction of BHs is typically much higher than what was previously thought. The $\mathrm{BH}$ dynamics near the cluster center leads to dynamical formation of new binaries and dynamical ejections, and acts as a persistent and significant energy source for these clusters. We have started exploring effects of BHs on the global evolution and survival of star clusters. We find that the evolution as well as survival of massive star clusters can critically depend on the details of the initial assumptions related to $\mathrm{BH}$ formation physics, such as natal kick distribution, and the initial stellar mass function (IMF). In this article we will present our latest results.
\end{abstract}

Keywords. black hole physics-stellar dynamics-methods: n-body simulations-methods: numerical-globular clusters: general-kinematics and dynamics-galaxies: star clusters

\section{Introduction}

Massive star clusters, such as the GCs in the Milky Way (MW) and other galaxies are expected to form large numbers of BHs simply as a consequence of the IMF and the large initial numbers of stars $(N)$ they are born with. What happens to these BHs after formation, due to a combination of $\mathrm{BH}$ formation physics, such as birth-kicks in supernova $(\mathrm{SN})$, and overall dynamical evolution of the host cluster is a long standing unsolved question.

Following the classic work by Spitzer (1969), it was argued that the BHs, being more massive compared to typical cluster stars, will mass segregate on $\lesssim 10^{2} \mathrm{Myr}$ timescales and form a compact subcluster that is dynamically decoupled from the rest of the cluster. Due to the small size, high density, and low (compared to the whole cluster) number of stars in this subcluster, frequent strong scattering interactions would eject most BHs from the cluster on $\sim$ Gyr timescales. As a result the old GCs were expected to contain at most a few BHs at their current old ages (Kulkarni et al. 1993, Sigurdsson et al. 1993, Portegies Zwart \& McMillan 2000, Kalogera et al. 2004). This understanding gained popularity because prior to 2012 all observed luminous X-ray sources (XRB) in the MW GCs were accreting neutron stars (NS; e.g., van Zyl et al. 2004, Lewin \& van der Klis 2006, Altamirano et al. 2010, Altamirano et al. 2012, Bozzo et al. 2011), consistent with no bound accreting BHs in today's GCs. 
This classical picture started to change with recent discoveries of candidate BHs in extragalactic as well as MW GCs (Maccarone et al. 2007, Irwin et al. 2010, Strader et al. 2012, Chomiuk et al. 2013). Interestingly, the GCs in the MW that contain the detected $\mathrm{BH}$ candidates, show large ranges in structural properties, indicating that presence of BHs in today's GCs may not be uncommon in general. Modern star-by-star, multiphysics simulations also find that $\mathrm{BH}$ ejection is not nearly as efficient as was previously thought. The primary reason for this difference compared to the earlier understanding comes from the fact that the BH subclusters do not stay decoupled from the rest of the cluster for prolonged periods. The same interactions that dynamically eject BHs from the cluster, also expands the subcluster and the subcluster again dynamically couples with the rest of the cluster (Breen \& Heggie 2013, Morscher et al. 2015). As a result, the evaporation timescale for the $\mathrm{BH}$ subcluster increases dramatically, and even the old GCs may contain hundreds to thousands of BHs today. These simulations also find that only a small fraction of the bound $\mathrm{BHs}$ are binaries with one $\mathrm{BH}$ and one non- $\mathrm{BH}$ components. Thus, only a few such objects may become observable XRBs even when the actual number of bound BHs is high. Factoring in also the typical low duty cycles for these sources (Kalogera et al. 2004), detection of just a few BH candidates likely indicates the existence of a much larger number of BHs in these clusters (e.g., Strader et al. 2012; Umbreit 2012; Morscher et al. 2013, 2015).

In this article, we will focus on the effects of a large number of retained BHs on the global evolution of the host cluster. All past models usually made several initial assumptions related to the details of the BH-formation physics, and the IMF. Several of these so called standard assumptions are actually not well constrained from observations. We will explore the effects of changing some of these assumptions, namely the natal kick distribution for BHs, and the slope of the IMF $\left(d n / d M \propto M^{-\alpha_{1}}\right)$ for high-mass stars $\left(M>1 M_{\odot}\right)$ on the global evolution of the star cluster. In $\S 2$ we describe our numerical setup and assumptions. In $\S 3$ we present our key results. Finally, in $\S 4$ we conclude.

\section{Numerical Models}

We use a Hénon-type Monte Carlo code (Hénon 1971), CMC, developed and rigorously tested by Northwestern University's cluster dynamics group over the past decade (most recently, Fregeau \& Rasio 2007; Chatterjee et al. 2010, 2013; Umbreit et al. 2012; Pattabiraman et al. 2013). We follow the initial assumptions of Morscher et al. (2015) for our standard model (we call it $\mathrm{S}$ ). This model initially contains $N=8 \times 10^{5}$ stars, and overall binary fraction $f_{b}=0.05$. The initial positions and velocities of the objects (single stars and center of mass of the binary stars) according to a King profile with $w_{0}=5$ (King $1962,1965)$. The initial virial radius $r_{v}=2 \mathrm{pc}$. The cluster is at a galacto-centric distance $r_{\mathrm{GC}}=8 \mathrm{kpc}$. The primary masses are chosen from an IMF presented in Kroupa (2001) for cluster stars between 0.1 to $100 M_{\odot}$. We adopt their middle values for the slopes in all mass ranges for $\mathrm{S}$. The secondary masses are chosen assuming a uniform mass-ratio distribution between the primary mass and $0.1 M_{\odot}$. Initial binary periods $(P)$ are drawn from a uniform distribution in $\log P$, between 5 times the sum of the stellar radii to the initial local hard-soft boundary. Eccentricities are thermal. We choose metallicity $Z=0.001$. Single and binary stellar evolution is done using SSE and BSE (Hurley et al. 2000, 2002), updated to implement custom natal kicks for the NSs and BHs. All NSs are assumed to receive natal kicks drawn from a Maxwellian distribution with $\sigma_{\mathrm{NS}}=265 \mathrm{~km} \mathrm{~s}^{-1}$. We adopt a momentum-conserving kick prescription for the BHs, where the kick magnitude is calculated by first drawing from the same Maxwellian as the NSs, but then scaling this velocity by the fallback fraction (Fryer \& Kalogera 2001, Belczynski et al. 2002). 

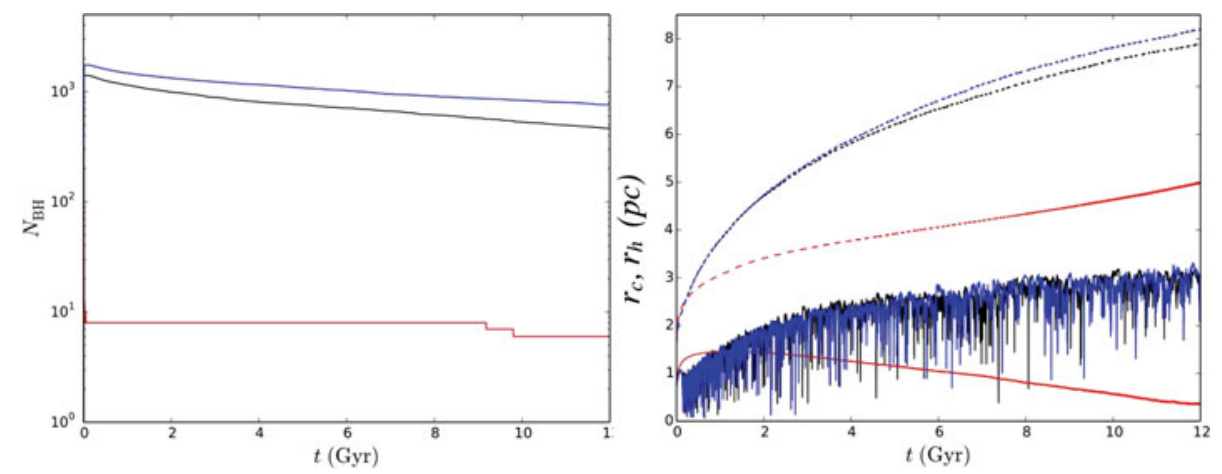

Figure 1. Evolution of the number of bound BHs in the cluster (left), and their structural properties, $r_{c}$, and $r_{h}$ (right), for models S (black), K1 (red), and K0.1 (blue). The solid and dotted lines on the right panel denote $r_{c}$ and $r_{h}$, respectively, for each model.

For example, if fallback fraction is $x_{\mathrm{FB}}=1$, i.e., no mass is lost during $\mathrm{SN}$, the kick magnitude is zero.

While it is widely accepted that the NSs receive large natal kicks, the distribution of natal kicks for BHs is not well constrained either via observations or via theory. Detailed analysis of kick distributions of individual BH X-ray binaries result in widely varying constraints on kick velocities between these systems (e.g., Brandt et al. 1995; Nelemans et al. 1999; Willems et al. 2005; Gualandris et al. 2005; Dhawan et al. 2007; Fragos et al. 2009; Wong et al. 2012, 2014). On the other hand, recently, Repetto et al. (2012) suggested that the position offsets of the BH XRBs from the Galactic plane indicates that the natal kicks from BHs can be as high as NSs. Furthermore, they found that there is no clear correlation between the BH mass and its inferred kick magnitude, which would be expected if they all received momentum-conserving natal kicks based on $x_{\mathrm{FB}}$. Modeling of core-collapse SN by Pejcha \& Thompson (2015) also suggests that the natal kicks are not related in a simple way to the mass of the BHs. Clearly, the issue of natal kicks for BHs is far from settled. We simulate three additional models by changing our initial assumptions of the natal kick distribution for the BHs. In these models we assume that the natal kicks for the BHs are not mass-dependent. Instead, they receive kicks from a Maxwellian with $\sigma_{\mathrm{BH}}=f \times \sigma_{\mathrm{NS}}$, where $f$ is a constant scaling fraction. We simulate two models with $f=1$ and 0.1 , and call these models K1 and K0.1, respectively. All other assumptions are exactly the same as in S.

The choice of IMF, especially $\alpha_{1}$, significantly affect the relative abundance of the high-mass stars, and as a result, the number of BHs a cluster with some given initial $N$ would form. While, the middle values for the slopes presented in Kroupa (2001) are commonly adopted for most simulations, they have large $1 \sigma$ errorbars, e.g., $\alpha_{1}=2.3 \pm 0.7$. We simulate two other models by changing $\alpha_{1}$ to 1.6 and 3 , and call these models I-1.6 and $\mathrm{I}-3$, respectively. Our model S of course already adopts $\alpha_{1}=2.3$.

\section{Results}

In our model $\mathrm{S}, \approx 2 \times 10^{3} \mathrm{BHs}$ are initially formed. The BHs drive repeated core-collapse episodes (Fig. 1). The core re-expands via dynamical binary formation as well as $\mathrm{BH}$ ejections due to strong gravitational scattering encounters involving binaries. However, $\mathrm{BH}$ ejections are not nearly as efficient as what was previously suggested (Spitzer 1969). Based on the $x_{\mathrm{FB}}$-dependent, momentum-conserving kicks adopted in model S, about 400 $\mathrm{BHs}$ remain bound to the cluster at integration stopping time $t=12 \mathrm{Gyr}$. This result is 


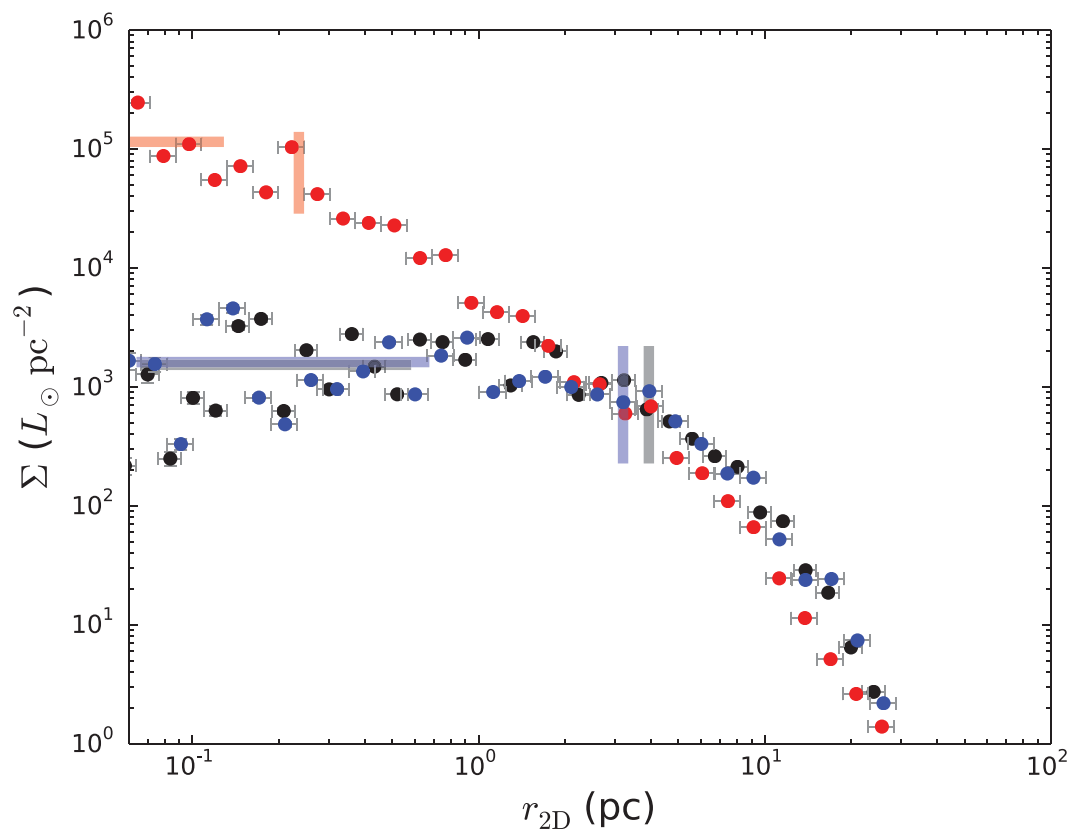

Figure 2. Surface brightness profiles at $t=12 \mathrm{Gyr}$ for models $\mathrm{S}$ (black), K0.1 (blue), and

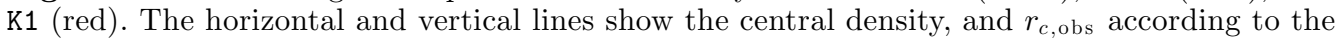
best-fit King profile. The surface brightness profile for K1, where most of the BHs were ejected due to large natal kicks, show a power-law rise of the density, typical of the so-called core-collapsed GCs in the MW. In contrast, the surface brightness profiles indicate rather large and low-density clusters for $\mathrm{S}$, and $\mathrm{K} 0.1$, where large numbers of BHs are retained until $t=12 \mathrm{Gyr}$.

in agreement with other recent star cluster models (e.g., Breen \& Heggie 2013; Morscher et al. 2015). The evolution of our model K0.1 is very similar to $\mathrm{S}$, except, K0.1 retains just shy of $10^{3}$ BHs at $t=12 \mathrm{Gyr}$.

We find that the distribution of natal kicks for the BHs can have a dramatic effect on the overall evolution of the host cluster. If the BHs receive kicks as large as the NSs, as is the case for our model $\mathrm{K} 1$, only $\approx 6 \mathrm{BHs}$ are retained in the cluster at late times. Most of the BHs are ejected from the cluster simply because of the kicks received during formation. Dynamical ejections at late times are insignificant. As a result, model K1's overall evolution is dramatically different from $\mathrm{S}$ and K0.1. In this case, since not many $\mathrm{BHs}$ are retained, the $\mathrm{BH}$-driven core-collapse episodes are unseen. In absence of the BH-driven energy source at the cluster center, $r_{c}$ starts contracting after about 1 Gyr. In comparison, $r_{c}$ keeps expanding until integration stopping time of $t=12$ Gyr due to $\mathrm{BH}$-mediated energy production at the cluster core for models $\mathrm{S}$, and KO.1.

The observable properties of the clusters are also significantly affected due to the difference in assumptions for the distribution of the BHs' natal kicks. For example, the surface brightness profiles (SBP) models S and K0.1 would indicate a rather puffy and low-density cluster, whereas, the SBP for model KO.1 would indicate a high-density core-collapsed cluster (Fig. 2). The only difference between the models is the persistent presence of the additional energy source from the hundreds of retained BHs in models $\mathrm{S}$ and K0.1, which is absent in model K1. Models $\mathrm{S}$ and K0.1 show central surface luminosity densities $\left(\Sigma_{c, \text { obs }}\right)$ between $10^{3}$ to $2 \times 10^{3} L_{\odot} \mathrm{pc}^{-2}$. The best-fit King core radii for these models are $r_{c, \text { obs }} \approx 4$, and $3 \mathrm{pc}$, respectively. In contrast, the surface luminosity density profile for model K1 show a power-law rise, typical of the core-collapse MW GCs 

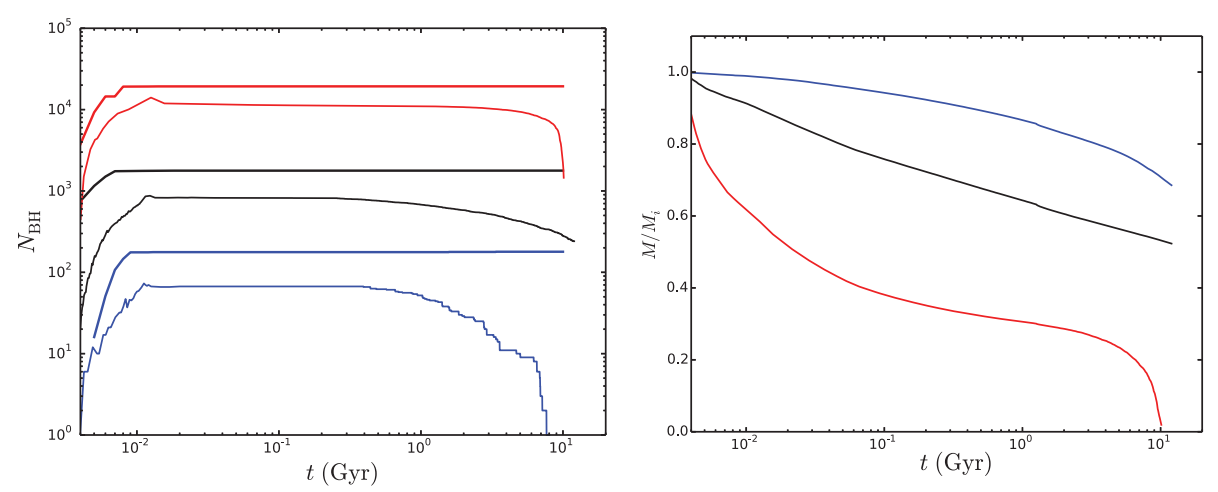

Figure 3. Left: Total number of BHs produced (thick lines) and retained (thin lines) in models I-1.6 (red), S (black), and I-3 (blue) as a function of cluster age. Right: Evolution of the cluster mass scaled by the initial mass of the cluster for models I-1.6 (red), S (black), and I-3 (blue). The model with the flat IMF slope, I-1.6, forms an order of magnitude higher number of BHs and dissolves within $\sim 8$ Gyr.

(e.g., Chatterjee et al. 2013). For K0.1, $\Sigma_{c, \mathrm{obs}} \sim 8 \times 10^{4} L_{\odot} \mathrm{pc}^{-2}$, and $r_{c, \mathrm{obs}}=0.2 \mathrm{pc}$. The half-light radii for models $\mathrm{S}, \mathrm{KO} .1$, and $\mathrm{K} 1$ are $r_{\mathrm{hl}}=5.5,3.4$, and $2.5 \mathrm{pc}$, respectively.

Similar to the distribution in natal kicks for the BHs, the IMF for high-mass stars also significantly affect the evolution of the clusters. Model I-1.6, with an adopted $\alpha_{1}=1.6$ (see $\S 2$ ) forms $\sim 2 \times 10^{4} \mathrm{BHs,}$ almost an order of magnitude more than in model S. The high amount of mass loss from stellar winds, compact object formation from the much higher number of high-mass stars, as well as the efficient energy source from the large number of BHs at the cluster's center leads to an eventual dissolution of this cluster after about 8 Gyr (Fig. 3). This is consistent with the predictions of the classic study by Chernoff \& Weinberg (1990). We also find that a model with such a flat IMF for highmass stars would always dissolve, no matter how low the BH natal kicks are. However, significantly different and lower wind mass loss (e..g, Vink et al. 2001) may result in clusters that survive longer. In model I-3, only about $200 \mathrm{BHs}$ are formed. All of these $\mathrm{BHs}$ are ejected from the cluster within $8 \mathrm{Gyr}$. Devoid of bound $\mathrm{BHs}, r_{c}$ of model I-3 starts decreasing. At $12 \mathrm{Gyr}$, for I-3, $r_{\mathrm{hl}}=1.2 \mathrm{pc}, r_{c, \mathrm{obs}}=0.15 \mathrm{pc}$, and central surface density is $4 \times 10^{5} L_{\odot} \mathrm{pc}^{-2}$.

\section{Discussion}

In this article we report results from an ongoing study that investigates the effects of the initial assumptions related to $\mathrm{BHs}$ on the overall evolution of the host cluster. Here we present results from two types of variations from a standard model that adopts commonly assumed initial properties. We change the distribution of natal kicks for the $\mathrm{BHs}$ and find that starting from clusters that are otherwise identical, variations in this assumption can dramatically alter the evolution of the cluster. For example, if the BHs receive birth kicks as large as the NSs, most $\mathrm{BHs}$ are ejected from the cluster and the cluster, at $12 \mathrm{Gyr}$, appears as a dense core-collapsed GC. On the other hand, if the BHs do not receive large natal kicks, hundreds of $\mathrm{BHs}$ may remain bound to the cluster till 12 Gyr, however, in these cases, the resulting cluster appears as puffy, low-density GCs.

Similar to the natal kicks, the power-law index of the IMF for stellar mass $M>1 M_{\odot}$, $\alpha_{1}$, plays a crucial role in determining the fate of a star cluster. We find that adopting $\alpha_{1}=1.6$, which is within the reported $1 \sigma$ errors presented in Kroupa (2001), results in 
clusters that dissolve within $\sim 8 \mathrm{Gyr}$ (model I-1.6). For $\alpha_{1}=2.3$ (the middle value of the Kroupa $2001 \mathrm{IMF}$; S), the cluster, at $12 \mathrm{Gyr}$ appears to be puffy, with a large $r_{c}$ and low $\Sigma_{c, \text { obs }}$. For $\alpha_{1}=3,(1 \sigma$ upper limit in Kroupa 2001; I-3), the cluster with otherwise identical initial properties, attain properties similar to the core-collapsed GCs in the MW.

\section{References}

Altamirano, D., Patruno, A., Heinke, C. O., Markwardt, C., et al. 2010 ApJ, 712, 58

Altamirano, D., Wijnands, R., Heinke, C. O., et al., 2012 The Astronomer's Telegram, 4264, 1 Belczynski, K. Kalogera, V., \& Bulik, T., 2002 ApJ, 572, 407

Bozzo, E., Ferrigno, C., Stevens, J., Belloni, T. M., Rodriguez, J., et al., 2011 A $\mathscr{S} A, 350,649$

Brandt, W. N., Podsiadlowski, P., \& Sigurdsson, S., 1995 MNRAS, 277, 35

Breen, P. G. \& Heggie, D. C., 2013 MNRAS, 432, 2779

Chatterjee, S., Fregeau, J. M., Umbreit, S., \& Rasio, F. A., 2010 ApJ, 719, 915

Chatterjee, S., Umbreit, S., Fregeau, J. M., \& Rasio, F. A., 2013 MNRAS, 429, 2881

Chernoff, D. F. \& Weinberg, M. D., 1990 ApJ, 351, 121

Chomiuk, L., Strader, J., Maccarone, T. J., Miller-Jones, J. C. A., et al., 2013 ApJ, 777, 69

Dhawan, V., Mirabel, I. F., Ribó, M., \& Rodrigues, I., 2007 ApJ, 668, 430

Fragos, T., Willems, B., Kalogera, V., Ivanova, N., Rockefeller, G., et al., 2009 ApJ, 697, 1057

Fregeau, J. M., \& Rasio, F. A., 2007 ApJ, 658, 1047

Fryer, C. L., \& Kalogera, V., 2001 ApJ, 554, 548

Gualandris, A., Colpi, M., Portegies Zwart, S., \& Possenti, A., 2005 ApJ, 618, 845

Hénon, M. H., 1971 Ap\&SSS, 14, 151

Hurley, J. R., Pols, O. R., \& Tout, C. A., $2000 M N R A S, 315,543$

Hurley, J. R., Tout, C. A., \& Pols, O. R., 2002 MNRAS, 329, 897

Irwin, J. A., Brink, T. G., Bregman, J. N., \& Roberts, T. P., 2010 ApJ, 712, 1

Kalogera, V., King, A. R., \& Rasio, F. A. 2004 ApJ, 601, 171

King, I., $1962 A J, 67,471$

King, I. R., $1965 A J, 70,376$

Kulkarni, S. R., Hut, P., \& McMillan, S. 1993 Nature, 364, 421

Lewin, W. H. G. \& van der Klis, M. 2006 Compact Stellar X-ray Sources, Ed. Walter Lewin \& Michiel van der Klis, Cambridge Astrophysics Series, No. 39. Cambridge, UK: Cambridge University Press, ISBN 978-0-521-82659-4

Maccarone, T. J., Kundu, A., Zepf, S. E., \& Rhode, K. L., 2007 Nature, 445, 183

Morscher, M., Umbreit, S., Farr, W. M., \& Rasio, F. A., 2013 ApJ, 763, 15

Morscher, M., Pattabiraman, B., Rodriguez, C., Rasio, F. A., \& Umbreit, S., 2015 ApJ, 800, 9

Nelemans, G., Tauris, T. M., \& van den Heuvel, E. P. J., 1999 A\&A, 352, 87

Pattabiraman, B., Umbreit, S., Liao, W.k., Choudhary, A., et al., 2013 ApJS, 204, 15

Pejcha, O., Thompson, T. A., 2015 ApJ, 801, 90

Portegies Zwart, S. F. \& McMillan, S. L. W. 2000 ApJ, 528, 17

Repetto, S., Davies, M. B., \& Sigurdsson, S., 2012 MNRAS, 425, 2799

Sigurdsson, S. \& Hernquist, L. 1993 Nature, 364, 423

Spitzer, Jr., L. 1969 ApJL, 158, 139

Strader, J., Chomiuk, L., Maccarone, T. J., Miller-Jones, J. C. A., et al., 2012 Nature, 490, 71

Umbreit, S., 2012 Nature, 490, 46

Umbreit, S., Fregeau, J. M., Chatterjee, S., \& Rasio, F. A., 2012 ApJ, 750, 31

van Zyl, L., Charles, P. A., Arribas, S., Naylor, T., Mediavilla, E., et al. 2004 MNRAS, 350, 649

Vink, J. S., de Koter, A., \& Lamers, H. J. G. L. M., 2001 A\&A, 369, 574

Willems, B., Henninger, M., Levin, T., Ivanova, N., Kalogera, V., et al., 2005 2005, 625, 324

Wong, T. W., Valsecchi, F., Fragos, T., \& Kalogera, V., 2012 ApJ, 747, 111

Wong, T. W., Valsecchi, F., Ansari, A., Fragos, T., Glebbeek, E., et al., 2014 ApJ, 790, 119 